\title{
The white dwarf cooling sequence of 47 Tucanae
}

\author{
Enrique García-Berro ${ }^{1,2}$, Santiago Torres ${ }^{1,2}$, Leandro G. Althaus ${ }^{3,4}$, and Marcelo M. Miller Bertolami ${ }^{4,5, \star}$ \\ 1 Departament de Física Aplicada, Universitat Politècnica de Catalunya, c/Esteve Terrades 5, 08860 Castelldefels, Spain \\ e-mail: enrique.garcia-berro@upc.edu \\ ${ }^{2}$ Institute for Space Studies of Catalonia, c/Gran Capita 2-4, Edif. Nexus 104, 08034 Barcelona, Spain \\ 3 Facultad de Ciencias Astronómicas y Geofísicas, Universidad Nacional de La Plata, Paseo del Bosque s/n, 1900 La Plata, Argentina \\ 4 Instituto de Astrofísica de La Plata, UNLP-CONICET, Paseo del Bosque s/n, 1900 La Plata, Argentina \\ ${ }^{5}$ Max-Planck-Institut für Astrophysik, Karl-Schwarzschild-Strasse 1, 85748 Garching, Germany
}

Received 22 July 2014 / Accepted 1 October 2014

\begin{abstract}
Context. 47 Tucanae is one of the most interesting, well-observed, and theoretically studied globular clusters. This allows us to determine the reliability of our understanding of white dwarf cooling sequences, to compare different methods of determining its age, and to assess other important characteristics, such as its star formation history.

Aims. Here we present a population synthesis study of the cooling sequence of the globular cluster 47 Tucanae. In particular, we study the distribution of effective temperatures, the shape of the color-magnitude diagram, and the corresponding magnitude and color distributions.

Methods. To do this, we used an up-to-date population synthesis code based on Monte Carlo techniques that incorporates the most recent and reliable cooling sequences and an accurate modeling of the observational biases.

Results. Our theoretical models and the observed data agree well. Thus, our study disproves previous claims that there are still missing physics in the white dwarf cooling models at moderately high effective temperatures. We also derive the age of the cluster using the termination of the cooling sequence and obtain a good agreement with the age determinations made using the main-sequence turnoff. Finally, the star formation history of the cluster is compatible with that obtained using main-sequence stars, which predicts two distinct populations.

Conclusions. We conclude that a correct modeling of the white dwarf population of globular clusters, used in combination with the number counts of main -sequence stars, provides a unique tool to model the properties of globular clusters.
\end{abstract}

Key words. stars: luminosity function, mass function - globular clusters: general - globular clusters: individual: 47 Tucanae white dwarfs

\section{Introduction}

White dwarfs are the most common end of stellar evolution, and as such they convey important and valuable information about their parent populations. Moreover, their structure and evolutionary properties are well understood - see, for instance, Althaus et al. (2010) for a recent review - and their cooling times are, when controlled physical inputs are adopted, as reliable as mainsequence lifetimes (Salaris et al. 2013). These characteristics have allowed determining accurate ages using the termination of the degenerate sequence for both open and globular clusters. This includes, to cite a few examples, the old, metal-rich open cluster NGC 6791 (García-Berro et al. 2010), which has two distinct termination points of the cooling sequence (Bedin et al. 2005, 2008a,b), the young open clusters M 67 (Bellini et al. 2010) and NGC 2158 (Bedin et al. 2010), or the globular clusters M 4 (Hansen et al. 2002) and NGC 6397 (Hansen et al. 2013). However, the precise shape of the cooling sequence also carries important information about the individual characteristics of these clusters and can moreover help in determining the correctness of the theoretical white dwarf evolutionary sequences. Recently, some concerns - based on the degenerate cooling sequence of the globular cluster 47 Tuc - have been

\footnotetext{
* Post-doctoral Fellow of the Alexander von Humboldt Foundation.
}

raised about the reliability of the available cooling sequences (Goldsbury et al. 2012). 47 Tuc is a metal-rich globular cluster with metallicity $[\mathrm{Fe} / \mathrm{H}]=-0.75$ or, equivalently, $Z \approx 0.003$. Thus, accurate cooling ages and progenitor evolutionary times of the appropriate metallicity are available (Renedo et al. 2010). Hence, this cluster can be used as a testbed for studying the accuracy and correctness of the theory of white dwarf evolution. Estimates of its age can be obtained by fitting the mainsequence turnoff, yielding values ranging from $10 \mathrm{Gyr}$ to $13 \mathrm{Gyr}$ - see Thompson et al. (2010) for a careful discussion of the ages obtained from fitting different sets of isochrones to the mainsequence turn-off. Additionally, recent estimates based on the location of the faint turn-down of the white dwarf luminosity function give a slightly younger age of $9.9 \pm 0.7 \mathrm{Gyr}$ (Hansen et al. 2013). Here we assess the reliability of the cooling sequences using the available observational data. As we show below, the theoretical cooling sequences agree well with this set of data. After determining that the theoretical white dwarf cooling sequences agree with the empirical sequence, we determine the absolute age of 47 Tuc using three different methods and also investigate whether the recent determinations using number counts of main-sequence stars of the star formation history are compatible with the properties of the degenerate cooling sequence of this cluster. 


\section{Observational data and numerical setup}

\subsection{Observational data}

The set of data we employed is the set obtained by Kalirai et al. (2012), which was also employed later by Goldsbury et al. (2012) to perform their analysis. Kalirai et al. (2012) collected the photometry for white dwarfs in 47 Tuc using 121 orbits of the Hubble Space Telescope (HST). The exposures were taken with the Advanced Camera for Surveys (ACS) and the Wide Field Camera 3 (WFC3), and comprise 13 adjacent fields. A detailed and extensive description of the observations and of the data reduction procedure can be found in Kalirai et al. (2012), and we refer to their paper for additional details.

\subsection{Numerical setup}

We used an existing Monte Carlo simulator that has been extensively described in previous works (García-Berro et al. 1999; Torres et al. 2002; García-Berro et al. 2004). We therefore only summarize the ingredients that are most relevant for our work. Synthetic main-sequence stars are randomly drawn according to the initial mass function of Kroupa et al. (1993). The selected range of masses is that necessary to produce the white dwarf progenitors of 47 Tuc. In particular, a lower limit of $M>0.5 M_{\odot}$ guarantees that enough white dwarfs are produced for a broad range of cluster ages. In our reference model we adopted an age $T_{\mathrm{c}}=11.5 \mathrm{Gyr}$, consistent with the main-sequence turnoff age of 47 Tuc - see Goldsbury et al. (2012) and references therein. We also employed the star formation rate of Ventura et al. (2014), which consists of a first burst of star formation of duration $\Delta t=0.5 \mathrm{Gyr}$, followed by a short period ( $\sim 0.04 \mathrm{Gyr})$ during which the star formation activity ceased, and a second short burst of star formation that lasted for $\sim 0.06$ Gyr. During the first star formation burst, $25 \%$ of the white dwarf progenitors are formed, while the rest of the synthetic stars (75\%) - which have a helium enhancement $\Delta Y \sim 0.03$ - are formed during the second burst. According to Ventura et al. (2014), the initial firstpopulation in 47 Tuc was about 7.5 times more massive than the cluster current total mass. At present, only $20 \%$ of the stars belong to the population with primeval abundances (Milone et al. 2012). Consequently, there is a small inconsistency in the firstto-second generation number ratio employed in our calculations. Since we are trying to reproduce the present-day first-to-second generation ratio, this inconsistency might have consequences in our synthetic luminosity function. To check this, we conducted an additional set of calculations for which we varied the percentage of stars with primeval abundances by $5 \%$ and found that the differences in the corresponding white dwarf luminosity functions were negligible.

After we knew which stars had time to evolve to white dwarfs, we computed their photometric properties using the theoretical cooling sequences for white dwarfs with hydrogen atmospheres of Renedo et al. (2010). These cooling sequences are appropriate because the fraction of hydrogen-deficient white dwarfs for this cluster is negligible (Woodley et al. 2012). These evolutionary sequences were evolved self-consistently from the zero age main sequence (ZAMS), through the giant phase, the thermally pulsing asymptotic giant branch and mass-loss phases, and ultimately to the white dwarf stage, and encompass a wide range of stellar masses from $M_{\text {ZAMS }}=0.85$ to $5 M_{\odot}$. To obtain accurate evolutionary ages for the metallicity of 47 Tuc $(Z=0.003)$, we interpolated the cooling ages between the solar $(Z=0.01)$ and subsolar $(Z=0.001)$ values of

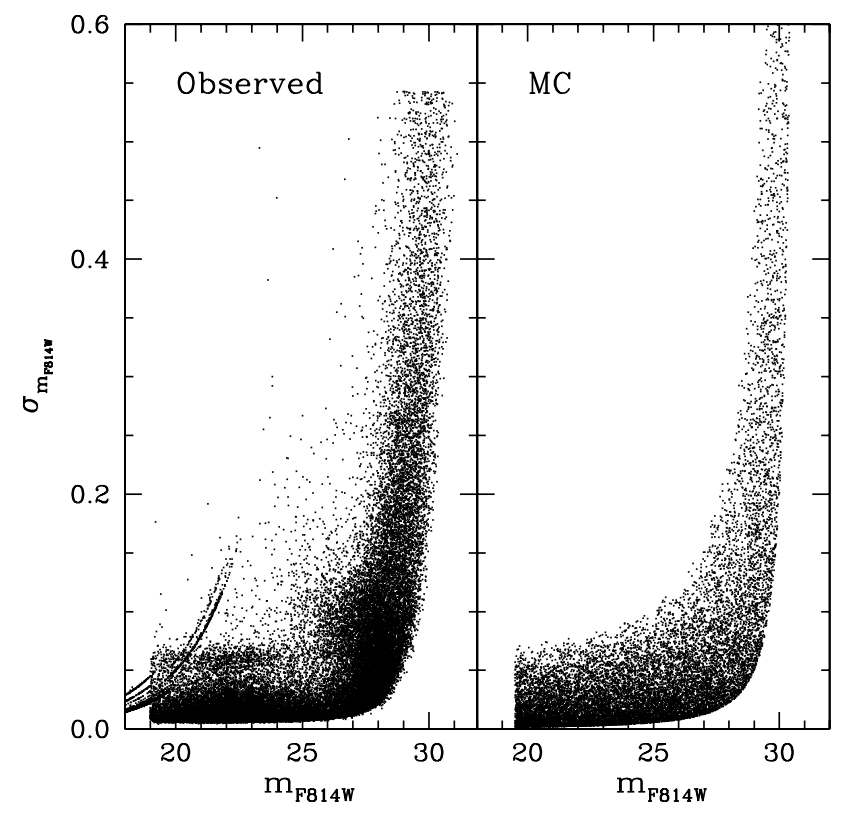

Fig. 1. Observed (left panel) and simulated (right panel) distributions of photometric errors. See text for details.

Renedo et al. (2010). For the second, helium-enhanced, population of synthetic stars we computed a new set of evolutionary sequences that encompass a broad range of helium enhancements. Finally, we also interpolated the white dwarf masses for the appropriate metallicity using the initial-to-final mass relationships of Renedo et al. (2010).

Photometric errors are assigned randomly according to the observed distribution. Specifically, for each synthetic white dwarf the photometric errors are drawn within a hyperbolically increasing band limited by $\sigma_{1}=0.2\left(m_{\mathrm{F} 814 \mathrm{~W}}-31.0\right)^{-2}$ and $\sigma_{\mathrm{u}}=1.7\left(m_{\mathrm{F} 814 \mathrm{~W}}-31.0\right)^{-2}+0.06$, which fits the observations of Kalirai et al. (2012) for the $F 814 W$ filter well. Specifically, the photometric errors are distributed within this band according to the expression $\sigma=\left(\sigma_{\mathrm{u}}-\sigma_{1}\right) x^{2}$, where $x \in(0,1)$ is a random number that follows an uniform distribution. Thus, the photometric errors increase linearly between the previously mentioned boundaries. Similar expressions were employed for the rest of the filters. The observed and simulated photometric errors of a typical Monte Carlo realization are compared in Fig. 1 for the $F 814 \mathrm{~W}$ filter. This figure shows that the observed and theoretically predicted distribution of errors agree reasonably well. In particular, the observed and theoretical distributions of the photometric errors have a relatively broad range of values for $F 814 \mathrm{~W}$, ranging from about $20 \mathrm{mag}$ to $25 \mathrm{mag}$, for which the width of the distribution remains almost flat. However, the width of the distribution increases abruptly for values of $F 814 \mathrm{~W}$ higher than this last value.

\section{Results}

\subsection{Empirical cooling curve}

First we discuss the distribution of white dwarf effective temperatures and compare it with the observed distribution, which is displayed in Fig. 2. Goldsbury et al. (2012) measured the effective temperatures of a large sample of white dwarfs in 47 Tuc. They produced a list sorted from the hottest to the coolest star to experimentally determine the rate at which white dwarfs are cooling. They assumed a constant rate for the white 
E. García-Berro et al.: The white dwarf cooling sequence of 47 Tuc

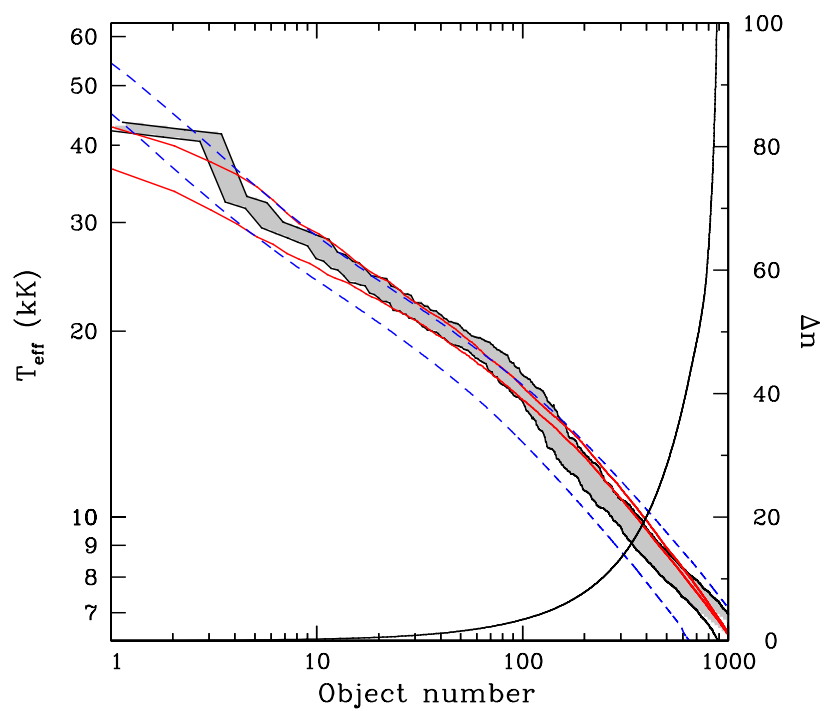

Fig. 2. Distribution of effective temperatures as a function of the white dwarf object number (left axis). The observational values of Goldsbury et al. (2012) are displayed in the gray shaded area, while the results of our Monte Carlo simulations are shown with red lines. The blue dashed lines show the cooling sequence for $M_{\mathrm{WD}}=0.53 M_{\odot}$ of Renedo et al. (2010) for $Z=0.001$ under different assumptions. The correction factor of the observed sample of white dwarfs is also displayed (right axis). See the online edition of the journal for a color version of this plot.

dwarf formation. Thus, the position in the sorted list is proportional to the time spent on the cooling sequence. Their sorted, completeness-corrected distribution of effective temperatures is shown Fig. 2 as a gray shaded band, which includes the $1 \sigma$ statistical errors. Also shown for illustrative purposes is the completeness correction (solid black line).

The most salient feature of the observed distribution is the pronounced break at effective temperatures $T_{\text {eff }}=20000 \mathrm{~K}$. This feature remained unexplained in Goldsbury et al. (2012) - see their Fig. 10 - and prompted them to attribute its origin to some missing piece of physics in all the existing models at moderate temperatures. The results of our population synthesis simulations for our reference model are shown as well and also include the $1 \sigma$ statistical deviations (upper and lower red lines). As can be seen, our calculations agree well with the observed data, without the need of invoking any additional physical mechanism in the cooling sequences, although they do not perfectly match the change in slope of the empirical cooling curve. In the following we separately discuss several possible reasons that may explain why our simulations agree better with the observed distribution than those of Goldsbury et al. (2012).

The first reason that explains why our model better fits the observed list is that we used updated main-sequence lifetimes, the initial-to-final mass relationship of Renedo et al. (2010) for the metallicity of the cluster, and interpolated cooling sequences for the precise metallicity of 47 Tuc, while Goldsbury et al. (2012) did not. All this results in a different turn-off mass for the cluster, and consequently in a different white dwarf mass at the top of the cooling sequence, hence in different cooling rates. To assess this, we compare in Fig. 3 the sorted list of Goldsbury et al. (2012) with the theoretical cooling sequences of two white dwarfs of masses $M_{\mathrm{WD}}=0.525 M_{\odot}$ and $0.520 M_{\odot}$, the white dwarf mass corresponding to the main-sequence turnoff of 47 Tuc, for two metallicities that embrace the metallicity of the cluster, $Z=10^{-3}$ and $Z=10^{-2}$, respectively. It is interesting to note that the $Z=10^{-3}$ sequence provides a

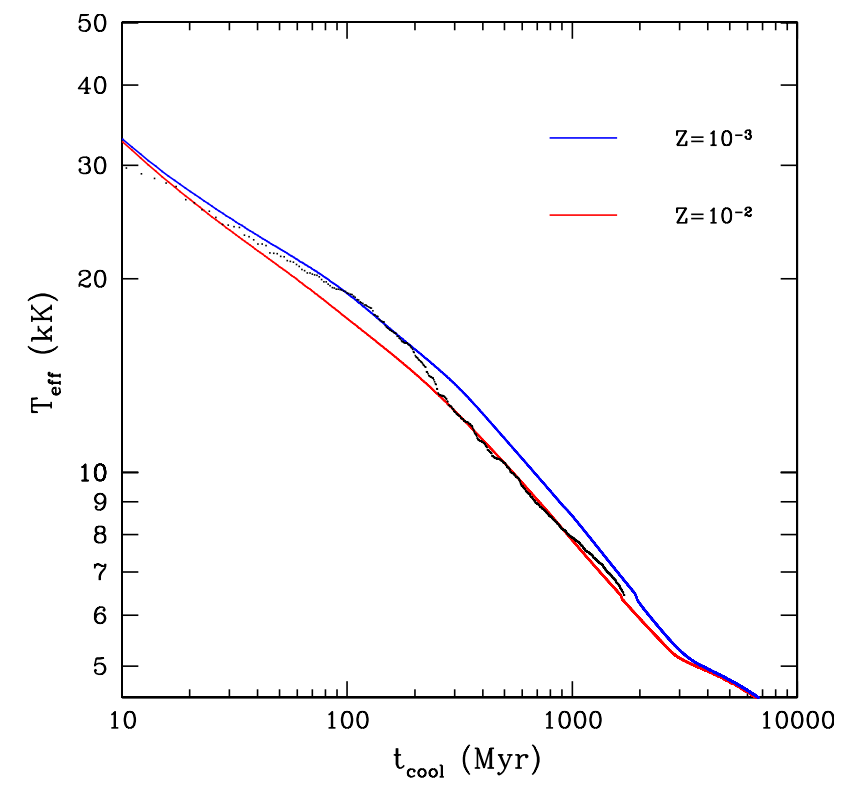

Fig. 3. Cooling sequences of Renedo et al. (2010) for the mass of the white dwarf corresponding to the main-sequence turn-off mass, and for two metallicities, compared with the empirical cooling sequence of Goldsbury et al. (2012).

good fit to the empirical cooling sequence of Goldsbury et al. (2012) for temperatures hotter than $T_{\text {eff }}=20000 \mathrm{~K}$, while the $Z=10^{-2}$ sequence is a good fit for temperatures colder than this value. Moreover, the theoretical cooling sequences bracket the observed distribution of effective temperatures, and therefore it is not surprising that our simulations fit better the observed distribution. Finally, another explanation for the better fit of our simulations to the observed distribution is that by construction, our simulations result in a spread of masses, while Goldsbury et al. (2012) adopted a single cooling track to compare with their observational data.

Nevertheless, although our simulations agree well overall with the observed distribution of effective temperatures, they do not fully reproduce the observed break in the empirical cooling curve at moderately high effective temperatures. We therefore explored the origin of this discrepancy. Clearly, metallicity cannot be at the origin of the break because in the region of interest the cooling sequences of the white dwarfs corresponding to the main turn-off mass run almost parallel. In addition, we adopted the star formation history of Ventura et al. (2014). We repeated our calculations employing a single burst of star formation, and the differences were found to be minor. Another possible origin for the break in the observed distribution may be the sudden increase in photometric errors for magnitudes larger than $25-$ see Fig. 1. We therefore checked whether the break in the distribution of observed errors is responsible for the observed break in the sorted list, and although including realistic photometric errors in the simulated population explains why our model is superior, we found that the observed break cannot be explained by the sudden increase in photometric errors for stars with magnitudes larger than 25 mag. Thus, the reason for the observed break in the empirical curve must be related to either the way the observed data were handled or to an unknown observational bias. We explore these possibilities next.

In Fig. 2 we also show the results of our simulations when we adopted the same procedure as used by Goldsbury et al. (2012). This is equivalent to adopting a single white dwarf cooling sequence of mass $M_{\mathrm{WD}}=0.53 M_{\odot}$ of a progenitor with metallicity 


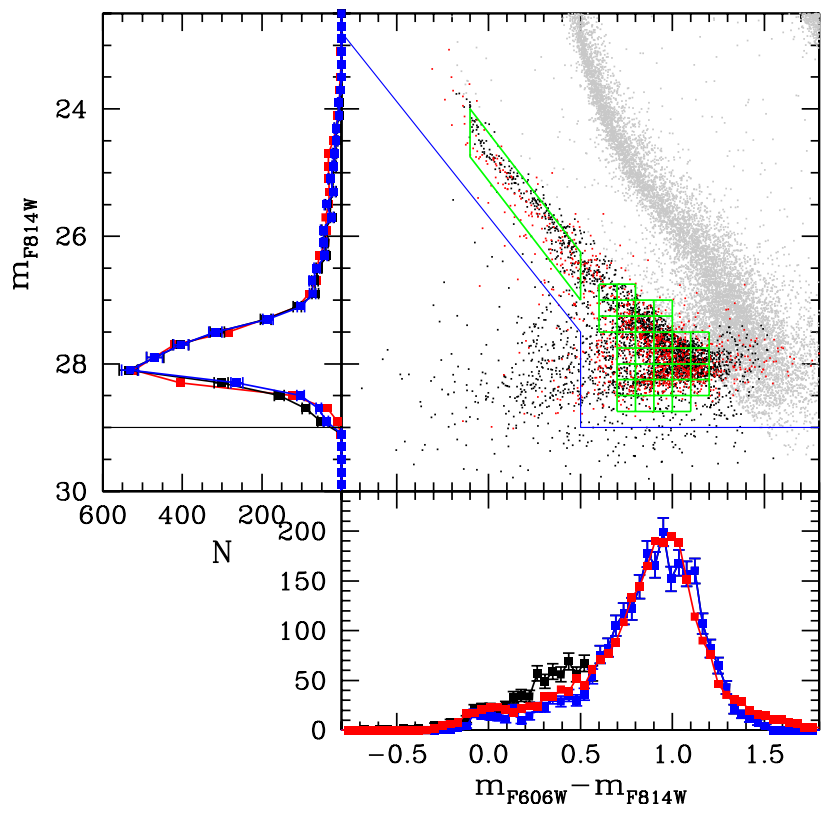

Fig. 4. White dwarf luminosity function, color-magnitude diagram, and color distribution of 47 Tuc. Gray dots represent observed main -sequence stars, black dots correspond to white dwarfs observed in the field, while red points denote the results of our synthetic population of white dwarfs. The green squares represent the regions of the color-magnitude for which the $\chi^{2}$ test was performed, while the blue thin lines correspond to the cuts adopted to compute the distributions. The red curves correspond to the simulated distributions obtained when no cuts are adopted, the blue curves are the observed distributions computed using our cuts, while the black lines are the observed distributions when no cuts are employed. See the online edition of the journal for a color version of this figure and the main text for additional details.

$Z=0.001$. As can be seen, the first white dwarfs in the cooling curve have effective temperatures higher than $T_{\text {eff }} \sim 40000 \mathrm{~K}$, while in the sorted list of Goldsbury et al. (2012) none is found. If white dwarfs with $T_{\text {eff }} \gtrsim 40000 \mathrm{~K}$, which have very short evolutionary timescales, and hence are difficult to detect, are removed from the theoretical sorted list, the entire distribution is shifted toward the left in this diagram, and we obtain the lower blue dashed line. This simple experiment helps in solving the discrepancy found by Goldsbury et al. (2012), although it does not entirely remove the reported difference. To better assess this, we employed a Kolmogorov-Smirnov test of the cumulative distributions of effective temperatures. We first computed the statistic separation $D$, which measures the largest separation between the cumulative distribution of our simulations and the observed data. The statistical distances computed in this way are 0.0789 when the Monte Carlo simulation and the observed sorted list are compared, 0.2717 when the model obtained using the procedure of Goldsbury et al. (2012) is compared with the observed sorted list, and 0.2694 when from this last model the hottest white dwarfs are removed from the sorted list. Using these statistical distances, we computed the probability of the three models being compatible with the observed data. The probability of our Monte Carlo distribution being compatible with the observational one is $P \simeq 0.92$, while this probability decreases to $P \simeq 0.73$ when the procedure employed by Goldsbury et al. (2012) is adopted, independently of whether the hottest white dwarfs are removed from the sorted list or not. Thus, although in a strict statistical sense none of the models can be totally excluded to a significant level of confidence - for instance, $5 \%$ - our population synthesis model agrees better with the observed distribution of effective temperatures, and there is no reason to invoke a missing piece of physics at moderately high luminosities.

\subsection{Color-magnitude diagram}

After assessing the reliability of the theoretical cooling sequences, we now discuss the overall shape of the color-magnitude diagram, and the distributions of magnitudes and colors. All this information is displayed in Fig. 4. The central panel of this figure shows the observed stars and the synthetic white dwarfs. The distribution of synthetic stars perfectly overlaps that of observed stars, except at very low luminosities, for which a contamination from galaxies is very likely. For this reason, we introduced a magnitude cut at $m_{\mathrm{F} 814 \mathrm{~W}}=29$ mag and discarded these objects from the subsequent analysis. Additionally, since we do not have proper motions of the white dwarfs in 47 Tuc, we introduced two more cuts, which are also displayed in this figure. These cuts are also intended to discard all background objects that are not cluster members. In particular, we did not consider objects to the left of $F 606 W-F 814 W=0.5$ mag for magnitudes between $29.0 \mathrm{mag}$ and $27.5 \mathrm{mag}$, and objects to the left of the line $F 814 W=3.62((F 606 W-F 814 W)-0.5)+27.5$ for brighter magnitudes. Since the background contamination is also overimposed on the white dwarf cooling sequence, we also estimated the number of background contaminants still present in the green boxes in Fig. 4. We did this in a statistical way, by assuming that the density of contaminants within these regions is similar to that close to the exclusion line, and we found that the percentage of contamination of each of the green boxes in Fig. 4 is small, $\sim 3 \%$. We then computed the theoretical white dwarf luminosity function and compared it with the observed luminosity functions when no cuts were employed and when the color and magnitude cuts were used. Note that the differences between the two observed luminosity functions are negligible for magnitudes brighter than that of the peak at $28 \mathrm{mag}$, and very small for fainter magnitudes. Moreover, the agreement between theory and observations is again excellent. We emphasize that should some physics have been missing in the theoretical cooling tracks, we would not have been able to obtain such a good agreement at high luminosities. Finally, the bottom panel of this figure shows the color distributions. Again, the agreement is very good, except for the small bump in the observed distribution at $F 606 W-F 814 W \sim 0.3$ mag when no cuts are used. However, when we discard the sources that are very likely not cluster white dwarfs, the agreement is excellent.

As mentioned, the white dwarf cooling sequence of 47 Tuc carries interesting information about its star formation history and age. To derive this information, we used the following approach: we computed independent $\chi^{2}$ tests for the magnitude $\left(\chi_{\mathrm{F} 814 \mathrm{~W}}^{2}\right)$ and color $\left(\chi_{\mathrm{F} 606 \mathrm{~W}-\mathrm{F} 814 \mathrm{~W}}^{2}\right)$ distributions. Additionally, we calculated the number of white dwarfs inside each of the green boxes in the color-magnitude diagram of Fig. 4 - which are the same regions of this diagram as used by Hansen et al. (2013) to compare observations and simulations - and we performed an additional $\chi^{2}$ test, $\chi_{N}^{2}$. We then investigated the values of the several parameters that define the star formation history of the cluster - that is, its age, the duration of the two bursts, and their separation - that best fit the observed data, independently. That is, we sought the parameters of the star formation history of 47 Tuc that best fit either the white dwarf luminosity function, the color distribution, or the number of stars in each of the boxes in Fig. 4. Obviously, this procedure results in different values of the parameters that define the star formation history of 47 Tuc. 
Table 1. $\chi^{2}$ test of the luminosity function, color distribution, and color-magnitude diagram for different ages and durations of the first burst of star formation.

\begin{tabular}{|c|c|c|c|c|c|c|c|c|c|c|c|c|}
\hline \multirow{3}{*}{$T_{\mathrm{c}}(\mathrm{Gyr})$} & \multicolumn{4}{|c|}{$\chi_{\mathrm{F} 814 \mathrm{~W}}^{2} / \chi_{\min }^{2}$} & \multicolumn{4}{|c|}{$\chi_{\mathrm{F} 606 \mathrm{~W}-\mathrm{F} 814 \mathrm{~W}}^{2} / \chi_{\min }^{2}$} & \multicolumn{4}{|c|}{$\chi_{N}^{2} / \chi_{\min }^{2}$} \\
\hline & \multicolumn{12}{|c|}{$\Delta t(\mathrm{Gyr})$} \\
\hline & 0.25 & 0.50 & 0.75 & 1.0 & 0.25 & 0.50 & 0.75 & 1.0 & 0.25 & 0.50 & 0.75 & 1.0 \\
\hline 10.0 & 3.21 & 3.95 & 4.58 & 5.56 & 1.76 & 1.91 & 2.15 & 2.66 & 9.81 & 11.47 & 12.28 & 13.97 \\
\hline 10.5 & 1.96 & 2.44 & 2.97 & 3.57 & 1.29 & 1.44 & 1.63 & 1.81 & 5.93 & 7.67 & 9.22 & 10.46 \\
\hline 11.0 & 1.42 & 1.62 & 1.92 & 2.30 & 1.16 & 1.17 & 1.31 & 1.42 & 3.46 & 4.26 & 5.70 & 6.96 \\
\hline 11.5 & 1.25 & 1.27 & 1.33 & 1.42 & 1.00 & 1.00 & 1.03 & 1.10 & 1.49 & 2.08 & 2.95 & 3.81 \\
\hline 12.0 & 1.17 & 1.10 & 1.10 & 1.18 & 1.17 & 1.05 & 1.03 & 1.07 & 1.17 & 1.20 & 1.43 & 2.04 \\
\hline 12.5 & 1.53 & 1.35 & 1.13 & 1.00 & 1.59 & 1.39 & 1.14 & 1.11 & 1.49 & 1.31 & 1.00 & 1.19 \\
\hline 13.0 & 1.97 & 1.78 & 1.43 & 1.29 & 1.90 & 1.67 & 1.53 & 1.36 & 2.13 & 1.97 & 1.50 & 1.37 \\
\hline
\end{tabular}

Notes. We list the normalized value of $\chi^{2}$, that is, the value of $\chi^{2}$ over its lowest value.

The results of our analysis are shown in Table 1, where we list only the data for a reduced set of models in which we kept fixed the separation between the two bursts of star formation and the duration of the second burst, and varied the age of the cluster and the duration of the first burst. We remark that we nonetheless explored a significantly wider range of parameters, and that for the sake of conciseness we show here only a few models. The reason is that the values of $\chi^{2}$ are less sensitive to variations in the rest of parameters, and thus this set of models is quite representative. When the white dwarf luminosity function is employed to obtain the age of the cluster and the duration of the burst of star formation, the $\chi^{2}$ test favors an age $T_{\mathrm{c}} \simeq$ $12.5 \pm 1.0 \mathrm{Gyr}$ and a duration $\Delta t \simeq 1.0 \pm 0.5 \mathrm{Gyr}$. Instead, when the color distribution is employed, we obtain $T_{\mathrm{c}} \simeq 11.5 \pm 1.0 \mathrm{Gyr}$ and $\Delta t \simeq 0.6 \pm 0.5 \mathrm{Gyr}$, while the model that best fits the number of stars in each bin of the color-magnitude diagram has an age $T_{\mathrm{c}} \simeq 12.5 \pm 0.5 \mathrm{Gyr}$ and a duration of the burst of star formation $\Delta t \simeq 0.7 \pm 0.5 \mathrm{Gyr}$. These results agree with those of Ventura et al. (2014), agree with the absolute age determination of 47 Tuc using the eclipsing binary V69 (Thompson et al. 2010), and also agree with each other within the error bars.

\section{Conclusions}

We have assessed the reliability and accuracy of the available cooling tracks using the white dwarf sequence of 47 Tuc. We demonstrated that when the correct set of evolutionary sequences of the appropriate metallicity is employed, the photometric errors are treated correctly, and observational biases are taken care of, the agreement between the observed and simulated distributions of effective temperatures, magnitudes and colors, as well as the general appearance of the color-magnitude diagram is excellent, without the need of invoking any missing piece of physics at moderately high effective temperatures in the cooling sequences. While our models do not entirely reproduce the sudden change of slope in the empirical cooling sequence, it is worth noting that this change in the slope takes place in the region where the completeness correction factor becomes relevant. Thus it might be well possible that this feature only arises because of some unknown observational bias.

In a second phase, and given that we found no reason to suspect the theoretical cooling sequences are incomplete, we also used these distributions to study the age and star formation history of the cluster using three different distributions: the white dwarf luminosity function, the color distribution, and the number counts of stars in the color-magnitude diagram. Using these three methods, we obtained that the age of the cluster is
$T_{\mathrm{c}} \sim 12.0$ Gyr. Our results are compatible with the recent results of Ventura et al. (2014), who found that star formation in this cluster proceeded in two bursts, the first one of duration $\sim 0.4$ Gyr, while the second one lasted for $\sim 0.06 \mathrm{Gyr}$, separated by a gap of duration $\sim 0.04$ Gyr. We also found that the relative strengths of these bursts of star formation activity (25\% and $75 \%$ ), and a helium-enhanced population of white dwarf progenitors (born exclusively during the second burst) are also compatible with the characteristics of the white dwarf population.

Since our analysis of the cooling sequence of 47 Tuc closely agrees with what is obtained from studying the distribution of main-sequence stars, we conclude that a combined strategy provides a powerful tool that can be used to study other star clusters, and from this obtain important information about our Galaxy. In this sense, it is important to realize that Hansen et al. (2013) computed the age of NGC 6397, obtaining 12 Gyr, significantly longer than their computed age for 47 Tuc $(\sim 10 \mathrm{Gyr})$. This prompted them to suggest that there is quantitative evidence that metal-rich clusters like 47 Tuc formed later than the metal-poor halo clusters like NGC 6397. Our study indicates that 47 Tuc is older than previously thought, and consequently, although this may be true, more elaborated studies are needed.

Acknowledgements. This work was partially supported by MCINN grant AYA2011-23102 by the European Union FEDER funds, by AGENCIA through the Programa de Modernización Tecnológica BID 1728/OC-AR, and by PIP 112-200801-00940 grant from CONICET. We thank R. Goldsbury and B.M.S. Hansen for providing the observational data shown in Figs. 2 and 4.

\section{References}

Althaus, L. G., Córsico, A. H., Isern, J., \& García-Berro, E. 2010, A\&ARv, 18, 471

Bedin, L. R., Salaris, M., Piotto, G., et al. 2005, ApJ, 624, L45

Bedin, L. R., King, I. R., Anderson, J., et al. 2008a, ApJ, 678, 1279

Bedin, L. R., Salaris, M., Piotto, G., et al. 2008b, ApJ, 679, L29

Bedin, L. R., Salaris, M., King, I. R., et al. 2010, ApJ, 708, L32

Bellini, A., Bedin, L. R., Piotto, G., et al. 2010, A\&A, 513, A50

García-Berro, E., Torres, S., Isern, J., \& Burkert, A. 1999, MNRAS, 302, 173

García-Berro, E., Torres, S., Isern, J., \& Burkert, A. 2004, A\&A, 418, 53 García-Berro, E., Torres, S., Althaus, L. G., et al. 2010, Nature, 465, 194 Goldsbury, R., Heyl, J., Richer, H. B., et al. 2012, ApJ, 760, 78

Hansen, B. M. S., Brewer, J., Fahlman, G. G., et al. 2002, ApJ, 574, L155 Hansen, B. M. S., Kalirai, J. S., Anderson, J., et al. 2013, Nature, 500, 51 Kalirai, J. S., Richer, H. B., Anderson, J., et al. 2012, AJ, 143, 11 Kroupa, P., Tout, C. A., \& Gilmore, G. 1993, MNRAS, 262, 545 Milone, A. P., Piotto, G., Bedin, L. R., et al. 2012, ApJ, 744, 58 Renedo, I., Althaus, L. G., Miller Bertolami, M. M., et al. 2010, ApJ, 717, 183 Salaris, M., Althaus, L. G., \& García-Berro, E. 2013, A\&A, 555, A96

Thompson, I. B., Kaluzny, J., Rucinski, S. M., et al. 2010, AJ, 139, 329

Torres, S., García-Berro, E., Burkert, A., \& Isern, J. 2002, MNRAS, 336, 971 Ventura, P., Criscienzo, M. D., D'Antona, F., et al. 2014, MNRAS, 437, 3274 Woodley, K. A., Goldsbury, R., Kalirai, J. S., et al. 2012, AJ, 143, 50 